\title{
ESTILOS DE AFRONTAMIENTO AL ESTRÉS EN PACIENTES DROGADEPENDIENTES
}

\author{
Gilmar Guevara Ortega \\ Héctor Hernández Valz \\ Tomy Flores Lezama
}

\begin{abstract}
RESUMEN
En la presente investigación se estudian los estilos de afrontamiento al estrés utilizados por pacientes drogadependientes que se encuentran recibiendo tratamiento en Comunidades Terapéuticas de Lima Metropolitana. La muestra está formada por 88 pacientes que tienen problemas de dependencia a sustancias psicoactivas como alcohol, pasta básica de cocaína, clorhidrato de cocaína y marihuana. El instrumento utilizado para la presente investigación es el Cuestionario COPE, elaborado por Carver, Scheiry Weintraub en 1989, este cuestionario permite evaluar las formas o estilos de afrontamiento que utiliza la gente ante eventos estresantes. El análisis psicométrico se realizó por medio de la correlación ítem-test corregida, implica que el ítem se compara con la prueba sin incluírsele. La consistencia interna se estableció utilizando el coeficiente de con fiabilidad Alfa de Cronbach.

Los resultados nos demuestran que los drogadependientes prefieren como estilos de afrontamiento: planificación, afrontamiento activo, postergación del afrontamiento y acudir a la religión. Asimismo se presentan como los estilos de menor preferencia, el desentendimiento conductual, la negación, la supresión de actividades competentes y el desentendimiento mental. También prefieren el estilo de afrontamiento que se centra en el problema y en último lugar de preferencia encontramos otros estilos de afrontamiento, que corresponden a las formas de desentendimiento conductual y mental principalmente. No se encontraron correlaciones significativas entre el tiempo de consumo y los estilos de afrontamiento, lo que nos lleva a afirmar que no hay ninguna diferencia entre el tiempo de consumo y los estilos de afrontamiento.
\end{abstract}

Palabras clave: Afrontamiento al estrés, drogadependencia, estrés, recaída, comunidad terapéutica.

\begin{abstract}
In the present investigation the confrontation styles are studied to the stress used by patient drug dependence that are receiving treatment in Therapeutic Communities of Metropolitan Lima. The sample is formed by 88 patients that have dependence problems to substances psychoactive like alcohol, pastures basic of cocaine, hydrochlorate of cocaine and marihuana. The instrument used for the present investigation is the Questionnaire COPE, elaborated by Carver, Scheir and Weintraub in 1989, this questionnaire allows to evaluate the forms or confrontation styles that people use before stressful events. The psychometric analysis was carried out by means of the correlation article-test corrected, it implies that the article is compared with the test without including. The consistency goes into it established using the coefficient of dependability Alpha of Cronbach.

The results demonstrate us that the drugdependence prefers as confrontation styles: planning, active confrontation, postponement of the confrontation and to go to the religion. Also is presented as the styles of smaller preference the behavioural disengagement, the negation, the suppression of competent activities and the mental disengagement. They also prefer the confrontation style that is centered in the problem and in I finish preference place we find other confrontation styles that correspond to the forms of behavioural disengagement and mental mainly. They were not significant correlations between the time of consumption and the confrontation styles, what takes us to affirm that there is not any difference between the time of consumption and the confrontation styles.
\end{abstract}

Words key: Confrontation to the stress, drugdependence, stress, relapse, therapeutic community. 
Uno de los objetivos principales al someter a un sujeto a tratamiento es el de conseguir que el adicto ejerza la abstinencia total, pudiendo además, lograrse un cambio en el estilo de vida. Sabiendo que en nuestro medio el estrés es parte de nuestra cotidianeidad, al vivir en un medio social en permanente crisis -y con crisis de todo tipo- las personas se encuentran bajo un estrés también permanente y de una u otra manera van minando su salud mental. Los drogadependientes han conseguido a través de su consumo compulsivo de drogas aliviar, en parte, su estrés; pero el mismo consumo también les va generando otro estrés del cual tendrán que "salir" consumiendo drogas. Se va instaurando un círculo negativo, en el cual se van hundiendo cada vez más.

Todo ello nos lleva a la necesidad de enseñar a la persona que se encuentra en tratamiento la propia utilización de recursos de afrontamiento positivos al estrés, pero para ello tenemos que conocer cuáles son los estilos de afrontamiento que usan los drogadependientes cuando no tienen su principal recurso de afrontamiento: la droga; en otras palabras, es necesario conocer cómo resuelven ellos sus eventos estresantes cuando no disponen de su "afronte químico".

Por ello es que intentamos conocer los principales estilos de afrontamiento que usan los drogadependientes en rehabilitación, para lo cual usaremos la prueba COPE creada por C.S. Carver y colaboradores.

La mayoría de los centros de tratamiento para las drogadependencia tienen como objetivos la abstinencia total y el desarrollo de un estilo de vida nuevo (Graña, J., 1994), sobre este último punto se entiende que aquí el rehabilitado tiene que aprender y utilizar formas adecuadas de afrontamiento a sus problemas que se le van apareciendo a medida que avanza en su reintegración social luego de su internamiento.

El estrés es una respuesta adaptativa de nuestro organismo ante demandas ambientales, caracterizado por una activación fisiológica bastante extendida, de duración variable y que de una u otra manera prepara al individuo para soportar tales eventos (Carlson 1996, Belloch 1995, Rosenzweig 1992, Caballo 1996). Esta definición es bastante extendida y muy usada, desde que Hans Selye adoptó y adaptó el término de la física clásica, para que sea usado por diversos profesionales de la salud, Selye entendía el estrés como una respuesta no específica del organismo (Belloch, 1995). Iniciándose su estudio en el marco de la fisiología, de donde parten dos conceptos básicos que servirán a Selye para construir su modelo de síndrome general de adaptación (SGA), medio interno, creado por Claude Bernard, padre de la medicina experimental, para referirse a los diferentes procesos fisiológicos que posibilitan la salud y la vida misma; Walter Cannon crea el término Homeostasis, para referirse a la constancia de nuestro medio interno ante cualquier variación de sus límites. En función de centrarse en cómo el cuerpo responde, es que los primeros modelos explicativos que se centran en la respuesta.

Otro grupo es constituido por las teorías que están basadas en el estímulo, dándole importancia a las variables ambientales o estresores, Belloch (1995), menciona a dos representantes de este modelo: T.H. Holmes y B. Sandían; recalcando su importancia en el desarrollo de los aspectos psicosociales y psicopatológicos del estrés.

Es Richard Lazarus, (1989), quien se sitúa entre ambos modelos y se ubica en medio de ambos para colocar allí a los procesos cognitivos. Es el iniciador de las teorías interactivas. Para ellas no son los tipos concretos de situación estresante, des-ligada de la persona lo que le interesa, sino el significado de la situación en tanto es percibida y evaluada por la persona. 
Lazarus establece que el estrés consta de tres procesos: la evaluación primaria, la evaluación secundaria y el afrontamiento. Durante la evaluación primaria el sujeto percibe y evalúa el evento estresante, aquí estarían presentes dos elementos claves, que como psicólogo ha tenido a bien contemplar: lo cognitivo y lo emocional. En la evaluación secundaria se elabora una respuesta a nivel mental, valorando los propios recursos para enfrentar la situación, esta respuesta debe atenuar o eliminar el evento estresante, y el afrontamiento sería el proceso de llevar a cabo esa respuesta. Este enfoque de Lazarus se diferencia sustantivamente de los demás en que le asigna un rol primordial a los aspectos cognitivos.(Buela-Casal, 1991).

El estilo de afrontamiento es la utilización de nuestros recursos personales para enfrentar las distintas situaciones estresantes, por ello es fácil presumir que existen ciertos factores disposicionales para que una persona haga frente de una manera determinada a dichos eventos. Entre las principales clasificaciones de estilos de afrontamiento tenemos: Byrne (1964) los clasifica como represor/sensibilizador, el primero tiende a la negación y evitación, el segundo es más vigilante y expansivo. Miller (1987) clasifica como incrementador (monitoring) y atenuador (blunting), el primero está alerta y sensibilizado respecto a la información relacionada con la amenaza, el segundo evita o transforma cognitivamente la información de amenaza. Actualmente Miller (1990) ha mejorado su modelo en función del tipo de situación estresante, ya sea en situaciones controlables e incontrolables. (citados por Belloch y Sandín, 1995; Caballo 1996).

Según Lazarus (1986) el afrontamiento como proceso implica lo siguiente:

1. El afrontamiento se emplea así el proceso sea adaptativo o inadaptativo, eficaz e ineficaz, es decir debe separarse el afrontamiento de sus resultados.

2. El afrontamiento depende del contexto.

3. Unas estrategias de afrontamiento son más estables o consistentes que otras.

4. El afrontamiento se puede centrar en el problema --cambiar la relación ambientepersona- y en la emoción implica cambiar el modo en que se trata o interpreta el problema para mitigar el problema-.

5. El afrontamiento depende de la evaluación respecto a que pueda o no hacerse algo para cambiar la situación.(citado por Belloch y Sandín, 1995)

Carver y Scheier (1994) --citado por Casuso, L., 1996- consideran que existen dos aspectos del afrontamiento:

Afrontamiento situacional.- El afrontamiento puede cambiar de un momento a otro a medida que va cambiando su relación con el entorno.

Afrontamiento disposicional.- Las personas desarrollan formas habituales de enfrentar las situaciones estresantes que se le presentan y que estas se toman en estilos que pueden influir en sus reacciones ante situaciones nuevas.

Muy pocos investigadores diferencian los estilos de las estrategias de afrontamiento. Establecer las diferencias pertinentes podría aclarar aspectos que se deben tomar en cuenta a la hora de realizar alguna intervención psicoterapéutica. «Los estilos de afrontamiento se refieren a predisposiciones personales para hacer frente a las situaciones y son los responsables de las preferencias individuales en el uso de unos u otros tipos de estrategia de afrontamiento, así como de su estabilidad temporal y situacional. Mientras que las estrategias de afrontamiento son los procesos concretos que se utilizan en cada contexto y pueden ser altamente cambiantes dependiendo de las condiciones desencadenantes» 
(Fernández-Abascal, 1997, citado por M.O. Martín, M.O. Jiménez y estímulo Fernández, 1997)

Una medición del afrontamiento como proceso debe:

1. Hacer referencia a pensamientos, sentimientos y actos específicos, y no a los informes de un individuo de 1 o que podría o quería hacer.

2. Ser examinada en un contexto específico.

3. Ser estudiada en diferentes períodos de tiempo.

\section{Afrontamiento y drogadependencia}

La drogadependencia es un problema de salud pública que en nuestro país alcanza niveles elevados. Los esfuerzos estatales y gubernamentales de nuestro país no se dan abasto ante la demanda, creciente día a día. Es por ello que surgen iniciativas privadas desde finales de los 80, son las Comunidades Terapéuticas (CT), que se circunscriben en un modelo de tratamiento alternativo al modelo médico imperante hasta entonces, enmarcado, como lo señala Rojas (1999). dentro del modelo /iluminativo, situación que nos llevaría a establecer un debate al respecto, pero que no es tema de la presente investigación.

El surgimiento de las CT marca el inicio de una forma de abordaje terapéutico que no es entendido y eso es hasta la actualidad- por los profesionales de la salud de ese entonces. Inclusive se ha tratado de poner "límites" a las CT al enmarcarlas dentro de una legislación que pretende convertirlas en centros asistenciales tradicionales, fruto de esas iniciativas es que muchas de las CT funcionan de manera bastante empírica, y prácticamente, fuera de la ley.

Desde nuestro medio social pueden llegar las primeras frustraciones que comiencen a debilitar su motivación. Sobre todo en una sociedad tan venida a menos en empleo, estudios, etc., puede surgir en ellos muchas veces la pregunta ¿Para qué me he rehabilitado? Justamente estas situaciones de crisis psicosocial determinan cuadros de estrés a los cuales el joven recurrirá muchas veces con la respuesta que le permite escapar y terminar todo esto, recurrirá a su modo de afrontamiento anterior: al consumo de alcohol y drogas.

Los factores de riesgo y factores protectores son importantes en el proceso de rehabilitación de los drogadependientes. Los factores de riesgo son aquellos eventos que influyen en el inicio de la conducta adictiva pero que también pueden provocar incidencia de consumo durante el proceso de tratamiento (Rojas, 1999) Los factores de protección modifican el impacto de los factores o situaciones estresantes disminuyendo el riesgo de aparición de un determinado trastorno. Su efecto es más global que los factores de riesgo y tienen mayor impacto en el bienestar en general. Los factores protectores son aquellos elementos que posibilitan una contención, y otorgan apoyo al individuo para que no recurra a acciones inadecuadas que puedan perjudicarlo. Cabe señalar que los factores de riesgo y protectores son eventos que ocurren en todas las esferas del comportamiento humano, así, pueden ser sociales, psicológicos, históricos, económicos, emocionales, biológicos, etc. Uno de estos probables factores protectores deberían ser los estilos de afrontamiento adecuados.

Un mismo factor puede ser de protección o de riesgo, dependiendo del contexto (por ejemplo, la raza o la religión).

Los drogadependientes y sus familias han encontrado en las CT un paliativo y quizás una solución a la problemática que enfrentan. Muchos de los drogadependientes que llegan a una CT lo hacen luego de varios años de consumo y en un grado de deterioro biológico, 
psicológico y social extremo. Por ello es que la intención de la presente investigación tratará de determinar si los mecanismos psicológicos que subyacen a los procesos de afrontamiento permanecen o han sido modificados por el consumo de drogas y a su vez determinar si el consumo de drogas posibilita un estilo de afrontamiento similar entre ellos.

Es obvio considerar que muchas veces la principal forma o estrategia de afrontamiento era recurrir a las drogas y/o alcohol para enfrentar los problemas. Al haber iniciado un tratamiento, y por consiguiente ya no consumir sustancias psicoactivas, deben desarrollar otras formas de afrontamiento que son necesarias de conocer por varias razones: a) ver si son las estrategias más adecuadas al perfil psicológico del sujeto; b) si el tipo de sustancia psicoactiva propicia o genera una forma determinada de afrontamiento; c) modificar los estilos de afrontamiento desadaptativos o perjudiciales al drogadependiente; d) prevenir las recaídas al posibilitar formas de afrontamiento adecua-das y eficaces, en otras palabras es hacer de sus modos de afrontamiento un factor protector.

\section{Definición de términos}

a) Afrontamiento: aquellos esfuerzos cognitivos y conductuales constantemente cambiantes que se desarrollan para manejar las demandas específicas externas y/o internas, que son evaluadas como excedentes o desbordantes de los recursos del individuo, (Lazarus y Folkman, 1984).

b) Estilos de afrontamiento: se refieren a predisposiciones personales para hacer frente a las situaciones, y son los responsables de las preferencias individuales en el uso de unos $\mathrm{u}$ otros tipos de estrategia de afrontamiento, así como de su estabilidad temporal y situacional.

c) Drogodependiente: se usa también el término dependiente a sustancias psicoactivas. La característica esencial consiste en un grupo de síntomas cognoscitivos, comportamentales y fisiológicos que indican que el individuo sigue consumiendo la sustancia a pesar de la aparición de problemas significativos relacionados con ella. Los síntomas de las dependencias son similares para todas las categorías de sustancias, pero con algunas de ellas los síntomas son menos patentes, e incluso pueden no aparecer (DSM IV, 1995).

\section{Otros estudios}

En 1996, Casuso (1996) realiza una adaptación del Cuestionario COPE (Carver, Séller y Weintraub, 1989) en estudiantes universitarios en dos Universidades, una estatal y la otra privada. En este trabajo realiza una modificación de las es-calas de la prueba, así también reagrupa los Ítems de la misma. Encuentra que los estudiantes universitarios utilizan, preferentemente, los estilos de afrontamiento de planificación, postergación del afrontamiento y afrontamiento activo; y los menos empleados por su muestra son acudir a la "religión, negación y desentendimiento conductual.

Escurra (1999) en un estudio con profesionales universitarios, con pluriempleo y empleo único; evaluó el patrón de conducta tipo A, una escala de apreciación del estrés laboral, el Cuestionario de los cinco grandes factores- BFQ el Inventario de solución y afrontamiento de problemas, y una Escala de autovaloración del desempeño laboral. Encontrando que los grupos de empleo único y pluriempleo alcanzan resultados similares en la solución y afrontamiento de problemas.

Canessa (2000), en su tesis universitaria realiza la adaptación psicométrica de la 
"Adolescent Coping Scale" de Erica Frydenberg (1993), compuesto por 80 Ítemes que conforman 18 escalas de diferentes formas de afrontamiento, que al final se agrupan en tres estilos básicos de afrontamiento: (A) Dirigido a resolver el problema, (B) afrontamiento no productivo y (C) Referencia a otros. Encontró que los adolescentes prefieren utilizar las estrategias: buscar diversiones relajantes, distracción física, preocuparse, fijarse en lo positivo y reservarlo para sí. Las estrategias menos empleadas fueron: reducción de la tensión, acción social, falta de afrontamiento, ignorar el problema y buscar ayuda profesional.

\section{METODOLOGIA}

\section{Tipo de estudio}

La presente investigación corresponde a un estudio del tipo descriptivo, pues se trata de conocer cuáles son los principales estilos y estrategias de afrontamiento que suelen llevar a cabo personas con problemas de drogadependencia.

\section{POBLACIÓN Y MUESTRA}

La población está conformada por personas drogadependientes varones que se encuentren recibiendo tratamiento ambulatorio o residencia en las diferentes Comunidades Terapéuticas de Lima Metropolitana.

La muestra corresponde a pacientes obtenidos por un muestreo no probabilístico, de tipo intencional de 3 Comunidades representativas de los conos sur, norte y centro de Lima.

El tamaño muestral se encuentra reducido debido a limitaciones de Índole administrativo que encontramos en estas instituciones.

\section{Descripción de la Muestra}

La muestra estuvo conformada por 88 drogadependientes varones que se encuentran siguiendo un tratamiento ambulatorio y residencial en Las comunidades Terapéuticas "Escuela de Vida", "Ayudémonos" y C.T. Sur. A continuación presentamos las principales características de la muestra.

Tabla 1

Distribución de la muestra según la comunidad Terapéutica de procedencia

\begin{tabular}{|l|c|c|}
\hline Comunidad & $\mathbf{N}^{\circ}$ de & $\boldsymbol{\%}$ \\
\hline Ayudémonos (Norte) & 26 & 29.5 \\
Escuela de Vida (centro) & 38 & 43.2 \\
C.T.Sur & 24 & 27.3 \\
\hline TOTAL & 88 & 100.0 \\
\hline
\end{tabular}

La Tabla 1 nos indica que la muestra estuvo conformada por integrantes de tres comunidades terapéuticas de Lima Metropolitana, distribuidos de acuerdo a los tres conos de Lima. El número de pacientes difiere en cada centro debido a que la población de los mismos es fluctuante y siempre se mantiene dentro de estos márgenes. 
Tabla 2

Distribución de la muestra según la edad de los pacientes

\begin{tabular}{|c|c|c|}
\hline Edad & $\mathbf{N}^{\circ}$ de & \% \\
\hline 18-22 años & 20 & 22,7 \\
23 -27 años & 13 & 14,8 \\
$28-32$ años & 13 & 14,8 \\
$33-37$ años & 15 & 17,0 \\
38 -42 años & 9 & 10,2 \\
$43-47$ años & 18 & 20,5 \\
\hline Total & 88 & 100 \\
\hline
\end{tabular}

Tabla 3

Distribución de la muestra de acuerdo al grado de instrucción

\begin{tabular}{|c|c|c|}
\hline Grado de Instruccción & $\mathbf{N}^{\circ}$ de Pacien. & \% \\
\hline Primaria incompleta & 1 & 1,1 \\
Primaria completa & 7 & 8,0 \\
Secundaria incompleta & 32 & 36,4 \\
Secundaria completa & 20 & 22,7 \\
Superior técnica & 6 & 6,8 \\
Superior universitaria & 22 & 25 \\
\hline Total & 88 & 100 \\
\hline
\end{tabular}

El grado de instrucción predominante se ubica en secundaria (incompleta y completa) siendo casi el $60 \%$ del total, y también es considerable el $25 \%$ de los que tienen educación universitaria.

\section{Tabla 4}

Distribución de la muestra según la sustancia principal de consumo

\begin{tabular}{|c|c|c|}
\hline Susto de CODS. & $\mathbf{N}^{\circ}$ de Pac. & \% \\
\hline Pasta Básic. Cocaína & 43 & 48,8 \\
Alcohol & 19 & 21,6 \\
Clorhidrat. de Coca. & 16 & 18,2 \\
Marihuana & 10 & 11,4 \\
\hline Total & 88 & 100 \\
\hline
\end{tabular}

Casi el 50\% de los drogadependientes evaluados presenta son dependientes a la PBC (pasta básica de cocaína), mientras que dependientes al alcohol constituyen el $21 \%$. Es interesante comprobar el aumento de dependientes al Clorhidrato de Cocaína que se encuentra en tratamiento.

\section{Tabla 5}

Distribución de la muestra de acuerdo al tiempo de consumo

\begin{tabular}{|c|c|c|}
\hline Tiempo de consumo & $\mathbf{N}^{\circ}$ de Pac. & $\%$ \\
\hline $0-1$ año & 1 & 1,1 \\
1 - 3 años & 9 & 10,2 \\
3 - 5 años & 11 & 12,5 \\
5-10años & 15 & 17,1 \\
10- 15 años & 16 & 18,2 \\
15 años a más & 36 & 40,9 \\
\hline Total & 88 & 100 \\
\hline
\end{tabular}

Aquí estamos considerando el tiempo transcurrido desde el primer contacto con la droga, de la cual son dependientes, los datos muestran que la mayoría de ellos, casi el $60 \%$ 
tienen más de 10 años de consumo. Esto es un indicativo del probable deterioro, psicológico, social y biológico, que se tiene que establecer para que los drogadependientes ingresen a tratamiento.

\section{INSTRUMENTO}

El instrumento utilizado para la presente investigación es el Cuestionario COPE, elaborado por Carver, Scheir y Weintraub en 1989, este cuestionario permite evaluar las formas o estilos de afrontamiento que utiliza la gente ante eventos estresantes. El cuestionario está compuesto de 52 ítemes que presentan cuatro alternativas de respuesta:

(1) Casi nunca hago esto

(2) A veces hago esto

(3) Usualmente hago esto

(4) Hago esto con mucha frecuencia

Presenta, además trece sub-escalas, correspondientes a los trece estilos de afrontamiento propuestos por los autores. Estas trece sub-escalas de estrategias de afrontamiento se pueden agrupar en tres formas o estilos más generales. Es importante señalar que Carver (1989) utiliza los términos estilo y estrategia de manera indistinta. La agrupación en función de los tres estilos de afrontamiento que utilizan los autores quedan como sigue:

A. Estilos de afrontamiento que se centran en el problema

1. Afrontamiento activo: $1,14,21,40$

2. Planificación: 2, $15,28,41$

3. Supresión de actividades competentes: $3,16,29,42$

4. Postergación del afrontamiento: 4, 17, 30,43

5. Búsqueda de apoyo social: 5,18,31,44 (por razones instrumentales) .

B. Estilos de afrontamiento que se centran en la emoción

6. Búsqueda de apoyo social: 6, 19,32, 45 (por razones emocionales)

7. Reinterpretación positiva y crecimiento: 7,20,33,46

8. Aceptación: $8,21,34,47$

9. Negación: $11,24,37,50$

10. Acudir a la religión: $9,22,35,48$

c. Otros estilos de afrontamiento

11.Enfocar y liberar emociones: 10, 23, 36, 49

12.Desentendimiento conductual : $12,25,38,51$

13.Desentendimiento mental: $13,26,39,52$

Como se observa cada una de las escalas está formada por cuatro ítemes, Casuso (1986), en una investigación de adaptación a la prueba, reagrupó los ítemes en el mismo número de escalas; en vista de que la población estudiada en nuestro país debe coincidir con la que se usará en la presente investigación, es que usaremos la REORGANIZACIÓN ya mencionada quedando las escalas de la siguiente manera:
A. Estilos de afrontamiento que se centran en el problema
1. Afrontamiento activo*: $14,27,40,42$
2. Planificación: $2,15,28,41$
3. Supresión de actividades competentes*: 3, 16, 29, 17 
4. Postergación del afrontamiento: 43, 30,46

5. Evadir el afrontamiento $* *: 33,52,4$

6. Búsqueda de apoyo social: $5,18,31,44$ (por razones instrumentales)

B. Estilos de afrontamiento que se centran en la emoción

7. Búsqueda de apoyo social: 6,19,32, 45 (por razones emocionales)

8. Aceptación: $8,21,34,47$

9.Negación: 7,11,20,24,37,50

10. Acudir a la religión: $9,22,35,48$

C. Otros estilos de afrontamiento

11. Enfocar y liberar emociones: 10, 23, 36,49

12. Desentendimiento conductual : $12,25,38,51$

13. Desentendimiento mental $*$ : $1,13,26,39$

* Estas escalas han cambiado algunos de sus Ítemes

** Esta escala es nueva, producto de la investigación de Casuso

*** La escala Reinterpretación positiva y crecimiento fue eliminada

El cuestionario COPE fue validado en una aplicación de estudiantes universitarios (Miami), las respuestas fueron sometidas al análisis factorial Que permitió obtener los factores principales utilizando la rotación oblicua, pues se hacia indispensable correlacionar los diferentes factores.

La confiabilidad del COPE se obtuvo utilizando la consistencia interna del Alfa de Cronbach.

\section{PROCEDIMIENTO}

El procedimiento de recogida de datos se hizo de forma colectiva, realizando previas coordinaciones con las comunidades terapéuticas seleccionadas, luego del permiso respectivo se visitaba la CT y se les explicaba la finalidad del estudio a los profesionales que laboraban allí. Una vez reunidos los pacientes se procedió a explicarles la prueba y se les iba asesoraba personalmente durante el desarrollo de la misma.

Los datos fueron luego procesados estadísticamente.

\section{RESULTADOS}

\section{Análisis psicométrico de la escala}

El análisis psicométrico se realizó por medio de la correlación ítem-test corregida, lo que implica que el Ítem se compara con la prueba sin incluírsele. La consistencia interna se estableció utilizando el coeficiente de confiabilidad Alfa de Cronbach. Obteniéndose los siguientes resultados:

Como podemos observar en la presente tabla los resultados demuestran que se han obtenido altos índices de confiabilidad, ya que el Alfa de Cronbach obtenida es alta en las escalas 7, 1, 3, 4, obteniéndose también cantidades importantes en las siguientes escalas con lo cual queda demostrada la con fiabilidad de la prueba. 
Tabla 6

Las escalas de afrontamiento y su coeficiente de confiabilidad

\begin{tabular}{|l|c|}
\hline \multicolumn{1}{|c|}{ Escalas } & Alfa de Cronbach \\
\hline 1. Afrontamiento activo & .7110 \\
2. Planificación & .5311 \\
3. Búsqueda de apoyo social (razones instrumentales) & .7195 \\
4. Búsqueda apoyo social (razones emocionales) & .7880 \\
5. Desentendimiento mental & .5086 \\
6. Aceptación & .5003 \\
7. Acudir a la religión & .8440 \\
8. Enfocar y liberar emociones & .5763 \\
9. Supresión de actividades competentes. & .5738 \\
10. Negación & .4613 \\
11. Desentendimiento conductual & .6006 \\
12. Evadir el afrontamiento & .4390 \\
13. Postergación del afrontamiento. & .5928 \\
\hline
\end{tabular}

Tabla 7

Ordenamiento de las escalas según preferencias

\begin{tabular}{|l|c|l|}
\hline \multicolumn{1}{|c|}{ Escalas } & Media & D.S. \\
\hline 1. Afrontamiento activo & 2.84 & .6186 \\
2.Planificación & 2.80 & .7404 \\
3. Postergación del afrontamiento & 2.76 & .6929 \\
4. Acudir a la religión & 2.75 & .9246 \\
5. Búsqueda de apoyo social (razones instrumentales) & 2.68 & .7050 \\
6. Búsqueda de apoyo social (razones emocionales) & 2.64 & .7887 \\
7. Aceptación & 2.60 & .6270 \\
8.Evadir el afrontamiento & 2.50 & .6511 \\
9. Enfocar y liberar emociones. & 2.45 & .6457 \\
10. Desentendimiento mental. & 2.28 & .6495 \\
11. Supresión de actividades competentes. & 2.22 & .6721 \\
12. Negación & 2.16 & .4851 \\
13. Desentendimiento conductual & 2.70 & .6124 \\
\hline
\end{tabular}

Aquí observamos las escalas de acuerdo a su preferencia, así tenemos que los drogadependientes prefieren como estilos de afrontamiento: planificación, afrontamiento activo, postergación del afrontamiento y acudir a la religión. Presentado como los estilos de menor preferencia el desentendimiento conductual, la negación, la supresión de actividades competentes y el desentendimiento mental.

Tabla 8

Preferencia en los estilos de afrontamiento agrupados

\begin{tabular}{|l|c|c|}
\hline \multicolumn{1}{|c|}{ Escalas } & Media & D.S. \\
\hline A. Estilos de afrontamiento que se centran en el problema & 2.63 & .48 \\
B. Estilos de afrontamiento que se centran en la emoción & 2.54 & .49 \\
C. Otros estilos de afrontamiento & 2.13 & .46 \\
\hline
\end{tabular}

La tabla nos muestra ahora a las escalas agrupadas en tres grandes estilos, vemos que los drogadependientes prefieren el estilo de afrontamiento que se centran en el problema y en último lugar de preferencia encontramos otros estilos de afrontamiento, que corresponden a las formas de desentendimiento conductual y mental principalmente. 
Tabla 9

Correlación entre tiempo y estilos de afrontamiento

\begin{tabular}{|l|c|}
\hline \multicolumn{1}{|c|}{ Escalas } & Correlación \\
\hline 1. Afrontamiento activo & -.006 \\
2.Planificación & -.006 \\
3.Búsqueda de apoyo social (razones instrumentales) & -.094 \\
4. Búsqueda de apoyo social (razones emocionales) & -.160 \\
5. Desentendimiento mental. & -.180 \\
6. Aceptación & .115 \\
7. Acudir a la religión & .019 \\
8. Enfocar y liberar emociones & .048 \\
9. Supresión de actividades competentes. & .093 \\
10. Negación & .032 \\
11. Desentendimiento conductual & .063 \\
12. Evadir el afrontamiento & -.097 \\
13. Postergación del afrontamiento & -.123 \\
\hline
\end{tabular}

Una de las correlaciones que realizaron fue entre el tiempo de consumo los estilos de afrontamiento, no encontrándose correlaciones significativas, lo que nos lleva a afirmar que no hay ninguna diferencia entre el tiempo de consumo y los estilos de afrontamiento.

Aquí correlacionamos el tiempo de abstinencia y los estilos de afrontamiento, encontrando correlaciones significativas en las escalas enfocar y liberar emociones y desentendimiento conductual, como podrá observarse estas correlaciones son negativas, lo que significa que a menor tiempo de abstinencia el sujeto usa más estos tipos de afrontamiento.

Tabla 10

Correlación entre tiempo de abstinencia y estilos de afrontamiento

\begin{tabular}{|l|c|}
\hline \multicolumn{1}{|c|}{ Escalas } & Correlación \\
\hline 1. Afrontamiento activo & .088 \\
2. Planificación & .130 \\
3. Búsqueda de apoyo social (razones instrumentales) & -.039 \\
4. Búsqueda de apoyo social (razones emocionales) & .040 \\
5. Dentendiemiento mental. & -.192 \\
6. Aceptación & -0.96 \\
7. Acudir a la religión & -.060 \\
8. Enfocar y liberar emociones & $-2.53^{*}$ \\
9. Supresión de actividades competentes. & -.029 \\
10. Negación. & .096 \\
11. Desentendimiento conductual & $-2.73^{*}$ \\
12. Evadir el afrontamiento & -.104 \\
13. Postergación del afrontamiento & .048 \\
\hline
\end{tabular}

Otro de los resultados hallados en cuanto a la diferencia entre tipo de droga consumida y estilos de afrontamiento es que se encontraron diferencias entre los consumidores a pasta básica de cocaína y alcohol en dos escalas: afrontamiento activo y supresión de actividades competentes. Los alcohólicos reportan una mayor preferencia por ambas formas de afronta-miento, cosa que es al contrario en los consumidores de PBC. En las demás es-calas de afrontamiento no se encontraron diferencias significativas respecto al cruce de ambas variables. 


\section{DISCUSIÓN}

La ubicación de las escalas de afrontamiento de acuerdo a la preferencia que demuestran los drogadependientes corrobora muchas de las presunciones clínicas observadas en el trabajo con individuo dependientes a sustancias psicoactivas. El uso preferente de planificación, afrontamiento activo, postergación del afrontamiento y acudir a la religión. Este último factor de acudir a la religión se ha mostrado siempre en los últimos lugares en poblaciones universitarias (Casuso, 1996) los otros tres restantes coinciden con las poblaciones estudiadas.

Quizás el factor religioso sea instaurado por el contexto espiritual, que es esencial en el tratamiento en la comunidades terapéuticas, o se podría suponer que debido al problema de la drogadependencia muchos adictos tratan de acudir a la religión como una forma de conseguir apoyo, y tratar de incorporar reglas de vida sanas y buscar círculos o redes sociales sanas.

Los dos estilos de afrontamiento que usan los drogadependientes coinciden por los encontrados por Casuso (1996), cabría suponer que las poblaciones sanas y las drogadependientes usan menos la negación y el desentendimiento conductual, podría ser que esta sea una norma en nuestra población.

También los drogadependientes usan preferentemente estilos de afrontamiento centrados en el problema, contra lo que se podría suponer, ya que en el trabajo con estas poblaciones ellos siempre parecen responder impulsivamente y se dejan llevar mucho por sus emociones, pero al menos pareciera que a la hora de enfrentar problemas, se centran en ellos y tratan de solucionar sin pensar en sus estados emocionales.

No se hallaron correlaciones significativas entre el tiempo de consumo y los estilos de afrontamiento, esta era una de las principales preocupaciones clínicas, ya que desde siempre se asume que el deterioro que produce la drogadependencia también es a nivel cognitivo-emocional. Pero los datos no le dan significación al cruce de estas dos variables, y eso que en la muestra hemos tenido gente con uno o dos años de consumo y drogadependientes con más de diez años de consumo.

En cambio si se han hallado correlación en dos escalas de afrontamiento y el tiempo de abstinencia. Hallándose una correlación negativa entre el tiempo de abstinencia y las escalas enfocar y liberar emociones y la de desentendimiento conductual. Esto significaría que los drogadependientes con menos tiempo de abstinencia prefieren usar más estas escalas que los que tienen mayor tiempo de abstinencia. Coincide esto con la asunción de responsabilidades, ya que el desentendimiento conductual es una forma de dejar de hacer algo para enfrentar un problema.

En cuanto a la diferencia entre los consumidores de diversas drogas sólo se hallaron diferencias entre los consumidores a pasta básica de cocaína y alcohol en dos escalas: afrontamiento activo y supresión de actividades competentes. Los alcohólicos reportan una mayor preferencia por ambas formas de afrontamiento, cosa que es al contrario en los consumidores de PBC. Este es un indicativo que siempre se maneja clínicamente, los alcohólicos tienen una forma y curso de deterioro más lento que los consumidores de PBC, al estar socialmente más integrados los alcohólicos afrontarían activamente sus problemas y suprimirían sus actividades competentes comparados con los adictos a la PBC.

\section{CONCLUSIONES}

1. El presente estudio demuestra que el cuestionario utilizado (COPE reorganizado) se 
considera un instrumento válido y confiable para usarlo en nuestro medio.

2. Los estilos de afrontamiento que prefieren usar los drogadependientes son: planificación, afrontamiento activo, postergación del afrontamiento y acudir a la religión.

3. Los estilos de afrontamiento de menor preferencia que usan los drogadependientes son: desentendimiento conductual, La negación, La supresión de actividades competentes y el desentendimiento mental.

4. La muestra de drogadependientes estudiada prefiere el estilo de afrontamiento que se centra en el problema, y en último lugar de preferencia encontramos otros estilos de afrontamiento, que corresponden a las formas de desentendimiento conductual y mental.

5. Encontramos que la forma de afrontamiento acudir a La religión es una de las preferidas por los drogadependientes, que comparadas con las poblaciones universitarias marca una diferencia sustancial, ya que éstos últimos la tienen entre las de menor preferencia.

6. No parecen existir diferencias entre las variables tiempo de consumo y estilos de afrontamiento.

7. Se han hallado diferencias entre el tiempo de abstinencia y el uso de los estilos de afrontamiento enfocar y liberar emociones.

8. Se hallaron diferencias entre los consumidores de alcohol y pasta básica de cocaína. Los alcohólicos usan más las formas de afrontamiento activo y supresión de actividades competentes.

\section{BIBLIOGRAFIA}

Belloch, A., Sandin, B., Manual de Psicopatología.McGraw-HilI /Interamericana de España Madrid, 1995

Buela-Casal, G., Caballo, V., Manual de psicología clínica aplicada. Siglo XXI editores. Madrid 1991

Caballo, V., Manual de psicopatología y trastornos psiquiátricos Vol II. Siglo XXI editores. Madrid 1996

Canessa, B., Adaptación de la prueba "Escala de afrontamiento para adolescentes" en escolares entre 14 y 17 años. Tesis de titulación Universidad de Lima. Lima 2000

Carlson, N. Fundamentos de Psicología fisiológica. Prentice- Hall Hispanoamericana. MéxicoDF,1996

Carver, C.S. Assesing coping strategies: A theoretical based approach. Journal of Personality and Social Psychology. 56,267-283. 1989

Casuso, L. Adaptación de la prueba COPE sobre estilos de afrontamiento en un grupo de estudiantes universitarios de Lima. Tesis PUCP,1996

DSM IV Manual diagnóstico y estadístico de los trastornos mentales, MASSON S.A. Barcelona, 1995

Escurra, M., El pluriempleo en profesionales universitarios. Revista Persona, 1999

Graña, J., Conductas adictivas: teoría evaluación y tratamiento. Editorial Debate. Madrid 1994

Lazarus, R., Folkman, S., Estrés y procesos cognitivos. Editorial Martínez Roca. Madrid 1986.

Nartin, M.D., Jiménez, MP. Fernández-Abasca, E. Estudio sobre la escala de estilos y estrategias deafrontamiento. Revista electrónica REME, 1998

Miller, W., Rollnick, S., II colloquio di motivazione. Centro Studi Erickson. Trento, 1994.

Rojas, M., Mujeres que consumen sustancias psicoactivas. CEDRO, Lima 1999.

Rosenzweig, M., Leiman, A. Psicología fisiológica. McGraw- Hill/ Interamericana de España. Madrid 1992. 